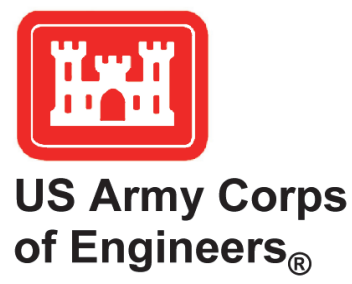

\title{
Historical Analysis of the Change in Percent Fines during Beach Nourishment
}

by Jennifer L. Coor and Jase D. Ousley

PURPOSE: This Coastal and Hydraulics Engineering Technical Note (CHETN) presents an analysis of the change in fine grained sediments (fines), comprised of silt and clay sized particles, content through hydraulic placement of sand on the beach through the dredging process during beach nourishment projects. The investigation sought to better understand the percentage of fines lost during hydraulic placement to provide greater confidence that sand sources with fines content greater than $5 \%$ are suitable for beach or nearshore placement.

This analysis, which utilizes sediments from projects within the State of Florida, may be used by the U.S. Army Corps of Engineers (USACE) and by regulatory agencies such as the Florida Department of Environmental Protection (FDEP) to guide decision making when designating dredged material for offshore disposal, nearshore, or beach placement, as part of navigational operation and maintenance projects $(\mathrm{O} \& \mathrm{M})$ and shore protection projects (SPP).

BACKGROUND: The loss of sand from Florida's beaches and coastal systems is a serious problem that affects not only the coastal system but also the economic livelihood of Florida's coastal communities. Beach erosion is a chronic problem in Florida, influenced by sea-level rise, natural coastal processes, storms, and inlet management (Clark 1993). The FDEP has documented 411.2 miles of critically eroded beaches, 8.7 miles of critically eroded inlet shoreline, 93.5 miles of non-critically eroded beaches, and 3.2 miles of non-critically eroded inlet shoreline statewide in a report eataloging the critically eroded beaches in Florida, as shown in Figure 1 (FDEP 2016a).

Florida's eroding beaches first began receiving sand as beneficial use from maintenance projects as early as the 1940s, but traditional beach nourishment practices did not begin until the early 1970s. Early beach nourishment projects focused on finding sufficient quantities of sand close to the project site, often not completing resource surveys in and around the sand sources or conducting a compatibility analysis with the native beach. In an effort to protect the environmental function and general character of the coastal system, the FDEP created the Sand Rule, Florida Administrative Code 62B-41.007(2)(j) and (k), in 1992 and amended the Rule in 2001, which specifies the criteria for sand placed on beaches within the State of Florida. The Sand Rule requires that material used for beach nourishment be similar in grain size distribution to the native or existing beach; additionally, fine sediment, defined by the percentage of material passing the \#230 U.S. Standard Sieve (silt and clay sized particles), must comprise 5\% or less of beach nourishment material. Material obtained from maintenance of navigation channels allows $10 \%$ fines or less for beneficial use/placement on the beach and $20 \%$ fines or less for beneficial use/placement in the nearshore. 
Identifying offshore sand resources suitable for future beach nourishment projects is critical to sustain Florida's beaches. Many beach quality sand resources located adjacent to the southeast Florida beaches have been exhausted (Ousley et al. 2014). Searching for sand sources with sufficient quantities of beach-compatible sand is becoming more difficult, time intensive, and cost prohibitive. In many locations it is becoming a difficult or impossible task to find sediment sources that meet the Sand Rule for beach nourishment, resulting in the need for more expensive truck-haul projects utilizing upland sand sources.

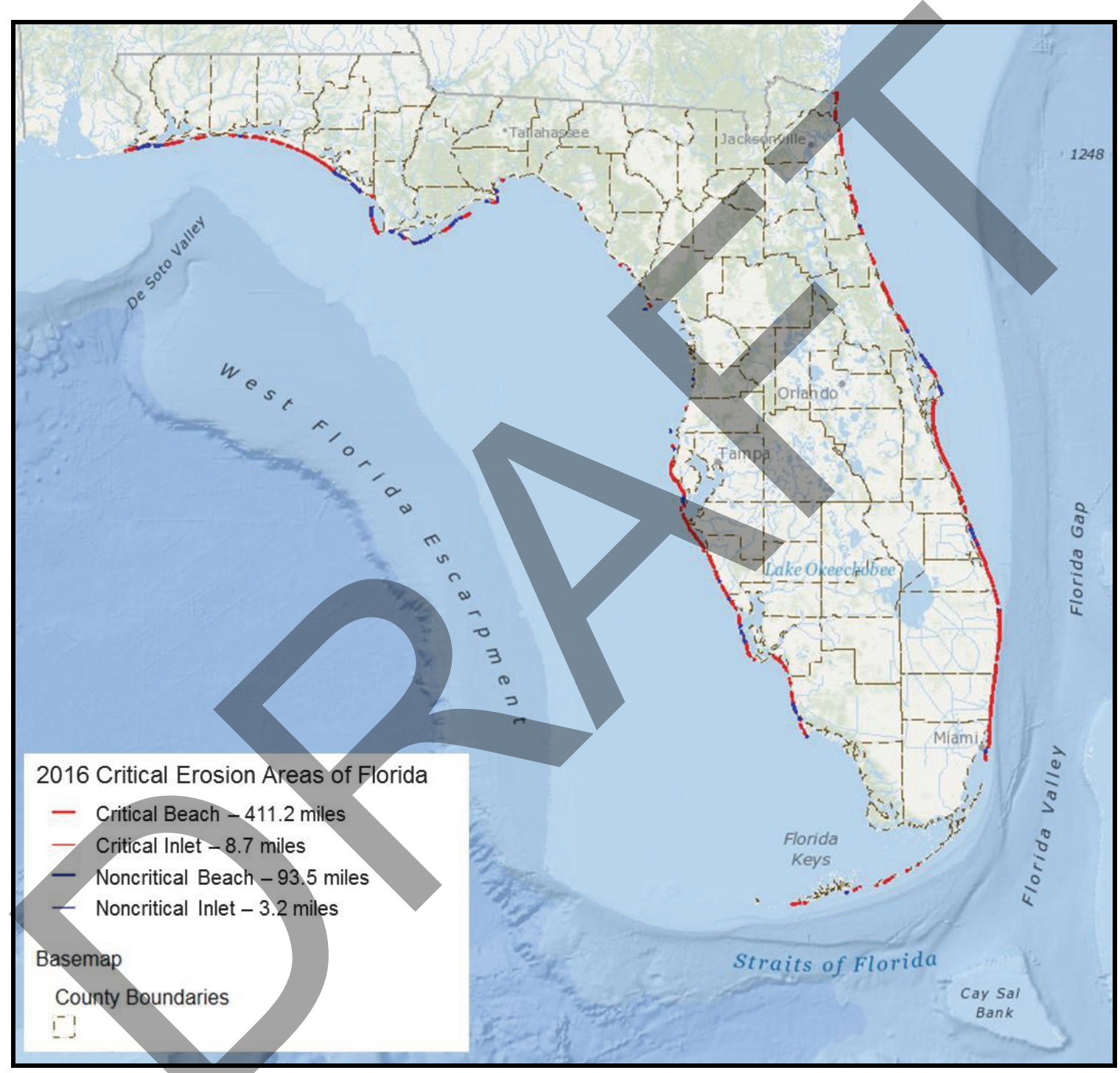

Figure 1. Statewide areas of critically and non-critically eroded shoreline. (Graphic modified from ROSSI database [FDEP 2016a].)

Historic project knowledge and data collected from sand sources (also referred to as borrow areas) and beach placement sites from around Florida indicate that fines are removed during dredging and beach placement on a variable, but consistent, basis. That is, the fines content of the dredged material is reduced during each phase of the dredging process, to include dredging the sand source, transport to the beach, and placement on the beach. Turbidity plumes, areas of suspended fine-grained sediment in the water column, are present both at the borrow site where 
dredging occurs, the overflow during transport (when using a hopper dredge), and at the beach placement site. Turbidity plumes are monitored as a permit condition on all projects; when the turbidity exceeds a determined threshold, dredging/construction activities must cease until there are fewer suspended sediments in the water column.

The present application of the Sand Rule criterion assumes zero reduction in the percent fines between the sand source and placement area, resulting in the inaccurate assumption that the fines content for the post-construction beach will be the same as the in situ value. This conservative assumption is understandable given the scarcity of data on fines loss predictability, but it has the negative effect of limiting, or completely eliminating, numerous potential sand sources with higher in situ fines contents than specified in the Sand Rule. The practice of regulating fines content based on in situ values also reduces the amount of coastal sediment that is being retained in the natural system during routine maintenance dredging events, as questionable material is frequently placed upland or disposed offshore, rather than used for beneficial purposes such as beach placement.

Comparing the in situ and post-construction samples only indicates the total change in the amount of fines during the entirety of the dredging project. This method of analysis cannot provide information about exactly where in the dredging process fines are removed or the impacts that the fines may have on sensitive marine resources.

METHODS: The 2014 Southeast Florida Sediment Assessment and Needs Determination (SAND) Study included a review of the change in fines content during dredging by comparing composite in situ sediment data and composite post-construction sediment data (Ousley et al. 2014). It was observed that in all cases fines were lost during the dredging process. This CHETN expands on the data set of in situ to post-construction change in fines initially presented in the SAND study (Ousley et al.2014) to compile a more robust data set for statistical analysis.

An FDEP Joint Coastal Permit (JCP) is needed to place sediment on any beach in the State of Florida. As part of the permitting process, the permittee must provide sediment data for the sand source and the existing, or native, beach placement area with a statistical comparison of the two demonstrating their similarity, referred to as a compatibility analysis. Following construction of a beach nourishment project, a JCP permittee must provide the FDEP with post-construction sediment data in the form of a compliance report to demonstrate that the sediment placed meets the established metrics outlined in the permit.

This work includes data from projects along the Atlantic and Gulf coasts of Florida (Figure 2), ranging from 1982-2015, compiled from the USACE and FDEP project file databases (Coor et al. 2015; FDEP 2016b). Composite in situ sediment data were obtained from historic compatibility analyses and geotechnical data found in FDEP JCP permit applications and/or construction quality assurance/quality control plans, referred to as QA/QC plans. Historical compliance reports were used to build the post-construction sediment data set. Data compiled for this analysis included project name, location, year constructed, in situ sand source grain size statistics, post-construction beach grain size statistics, and the method of dredging and placement (when available). Grain size data were calculated by the applicant/permittee of each project, and included the composite mean grain size and composite sorting coefficient (phi, $\Phi$ ), commonly determined by creating a weighted average for each sample area (Friedman and Sanders 1978; 
Tanner 1995; USACE 2001; Dean 2002); the composite percent of fines is the weighted average of the percent passing the \#230 US Standard Sieve for each sample area. The change in percent fines from the in situ sand source to the post-construction beach was used as the foundation for analysis (Equation 1).

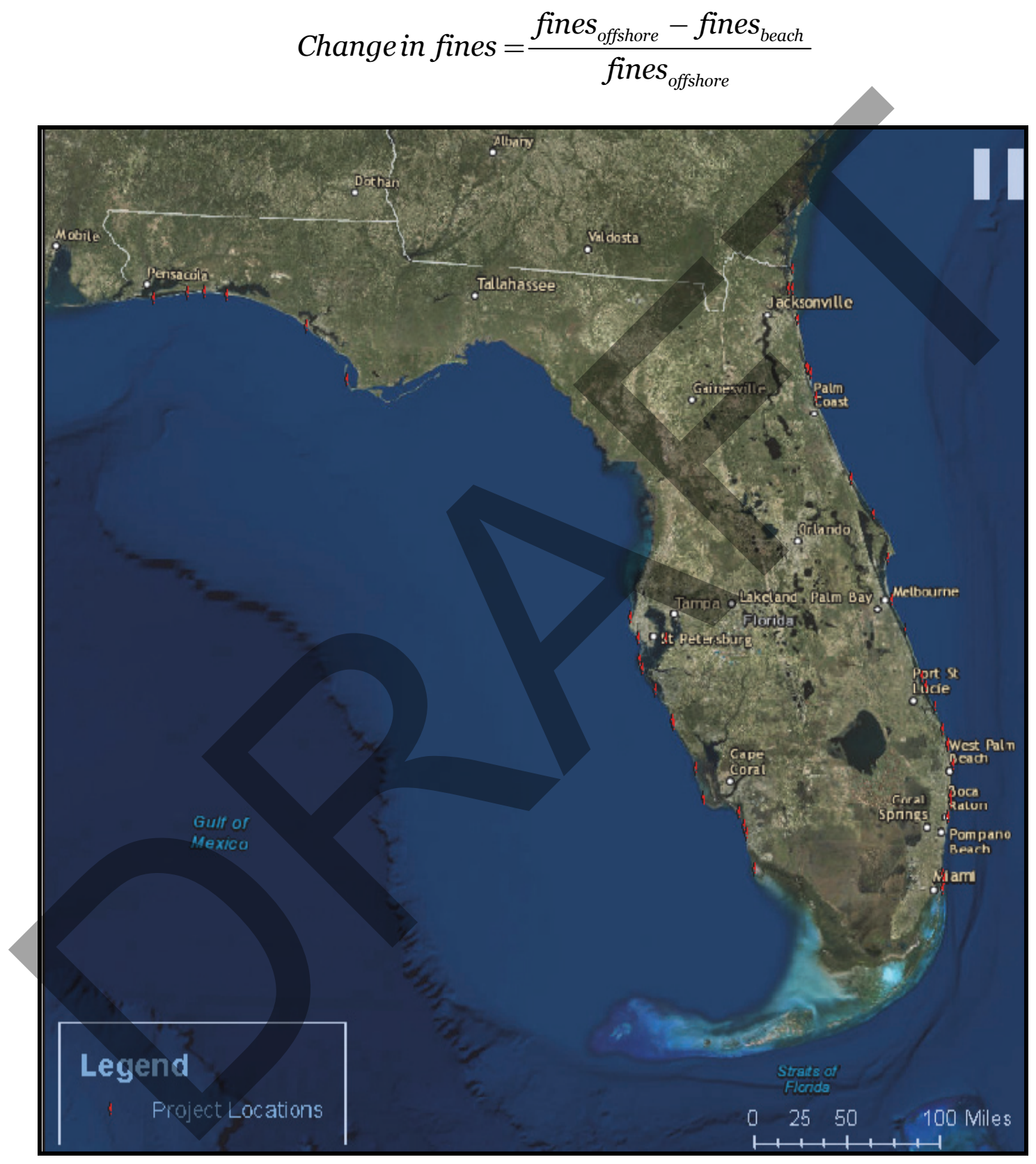

Figure 2. Map of Florida, highlighting project location sites sampled for fines loss in this study.

RESULTS: The following results discuss the overall loss of fines as determined from composite statistical calculations of the in situ sand source and the post-construction beach. The projects included in this study included beach nourishment projects utilizing offshore resources (to 
include offshore sand sources and inlet shoals) and beneficial use of material from maintenance dredging of navigation channels (to include the Intracoastal Waterway and inlet channels).

\begin{tabular}{|c|c|c|c|c|c|c|c|c|c|}
\hline \multirow[b]{2}{*}{$\begin{array}{l}\text { Project } \\
\text { Name }\end{array}$} & \multirow[b]{2}{*}{ County } & \multirow[b]{2}{*}{ Year } & \multirow[b]{2}{*}{$\begin{array}{c}\text { Fill } \\
\text { Volume } \\
\text { (cy) }\end{array}$} & \multirow[b]{2}{*}{$\begin{array}{l}\text { Sediment } \\
\text { Source }\end{array}$} & \multicolumn{2}{|c|}{$\begin{array}{c}\text { In Situ Sand } \\
\text { Source } \\
\text { Composite } \\
\end{array}$} & \multicolumn{2}{|c|}{$\begin{array}{l}\text { Post-Fill Beach } \\
\text { Composite }\end{array}$} & \multirow[b]{2}{*}{\begin{tabular}{|l} 
Percen \\
Change \\
in Fines
\end{tabular}} \\
\hline & & & & & $\begin{array}{l}\text { Mean } \\
(\mathrm{mm})\end{array}$ & \begin{tabular}{|c|}
$\%$ \\
Passing \\
$\# 230$ \\
\end{tabular} & $\begin{array}{l}\text { Mean } \\
(\mathrm{mm})\end{array}$ & \begin{tabular}{|c|}
$\%$ \\
Passing \\
$\# 230$ \\
\end{tabular} & \\
\hline $\begin{array}{c}\text { Lee SPP, } \\
\text { Captiva } \\
\text { Island }\end{array}$ & Lee & 1981 & 655,500 & Inlet & 0.2 & & & 0.5 & $75 \%$ \\
\hline $\begin{array}{c}\text { North Boca } \\
\text { Raton } \\
\text { Beach } \\
\text { Nourishment }\end{array}$ & Palm Beach & 1988 & $1,102,000$ & Offshore & & 0.51 & 0.35 & & $-96 \%$ \\
\hline $\begin{array}{l}\text { Broward } \\
\text { SPP, John } \\
\text { U Lloyd } \\
\text { Segment }\end{array}$ & Broward & 1989 & 603,423 & Offshore & & & 0.48 & 0.45 & $91 \%$ \\
\hline $\begin{array}{l}\text { Broward } \\
\text { SPP, } \\
\text { Hollywood/ } \\
\text { Hallandale }\end{array}$ & Broward & 1991 & $1,108,615$ & & 0.38 & & 0.32 & 5.3 & $-6 \%$ \\
\hline $\begin{array}{l}\text { Delray } \\
\text { Beach }\end{array}$ & Palm Beach & 1992 & $1,196,500$ & Offs & 0.29 & 0.6 & 0.26 & 0.67 & $-12 \%$ \\
\hline \multirow{2}{*}{\begin{tabular}{|c|} 
Longboat \\
Key Beach \\
Restoration \\
\end{tabular}} & \multirow[b]{2}{*}{ Pinellas } & \multirow[b]{2}{*}{1993} & \multirow[b]{2}{*}{$3,130,000$} & \multirow{3}{*}{ Beach } & 0.22 & 5 & 0.22 & 0.5 & $90 \%$ \\
\hline & & & & & 0.19 & 1.9 & 0.19 & 0.5 & $74 \%$ \\
\hline $\begin{array}{c}\text { Canaveral } \\
\text { Harbor - } \\
\text { Sand } \\
\text { Bypass } \\
\end{array}$ & & & 783,000 & & 0.28 & 5.3 & 0.11 & 4.23 & $20 \%$ \\
\hline $\begin{array}{c}\text { Bonita } \\
\text { Beach } \\
\text { Restoration }\end{array}$ & & 1995 & 217,000 & Inlet & 0.27 & 0.55 & 0.3 & 0.5 & $9 \%$ \\
\hline $\begin{array}{l}\text { Martin SPP, } \\
\text { Hutchinson } \\
\text { Island }\end{array}$ & Martin & 1996 & $1,340,000$ & Offshore & 0.29 & 3.5 & 0.63 & 0.5 & $86 \%$ \\
\hline $\begin{array}{c}\text { Sanibel } \\
\text { Island - Gulf } \\
\text { Pines Beach } \\
\text { Restoration }\end{array}$ & & 1996 & 220,000 & Offshore & 0.39 & 2.07 & 0.27 & 2.21 & $-7 \%$ \\
\hline $\begin{array}{l}\text { Lee SPP, } \\
\text { Captiva } \\
\text { Island }\end{array}$ & Lee & 1997 & 817,280 & Offshore & 0.39 & 2.07 & 0.35 & 2.44 & $-18 \%$ \\
\hline $\begin{array}{c}\text { Lido Key } \\
\text { Beach } \\
\text { Nourishment }\end{array}$ & Sarasota & 1998 & 292,500 & Inlet & 0.14 & 5 & 0.3 & 1.19 & $76 \%$ \\
\hline
\end{tabular}




\begin{tabular}{|c|c|c|c|c|c|c|c|c|c|}
\hline \multirow[b]{2}{*}{$\begin{array}{c}\text { Project } \\
\text { Name }\end{array}$} & \multirow[b]{2}{*}{ County } & \multirow[b]{2}{*}{ Year } & \multirow[b]{2}{*}{$\begin{array}{c}\text { Fill } \\
\text { Volume } \\
\text { (cy) }\end{array}$} & \multirow[b]{2}{*}{$\begin{array}{l}\text { Sediment } \\
\text { Source }\end{array}$} & \multicolumn{2}{|c|}{$\begin{array}{c}\text { In Situ Sand } \\
\text { Source } \\
\text { Composite } \\
\end{array}$} & \multicolumn{2}{|c|}{$\begin{array}{l}\text { Post-Fill Beach } \\
\text { Composite }\end{array}$} & \multirow[b]{2}{*}{$\begin{array}{l}\text { Percent } \\
\text { Change } \\
\text { in Fines }\end{array}$} \\
\hline & & & & & $\begin{array}{l}\text { Mean } \\
(\mathrm{mm})\end{array}$ & \begin{tabular}{|c|}
$\%$ \\
Passing \\
$\# 230$ \\
\end{tabular} & $\begin{array}{l}\text { Mean } \\
(\mathrm{mm})\end{array}$ & \begin{tabular}{|c|}
$\%$ \\
Passing \\
$\# 230$ \\
\end{tabular} & \\
\hline $\begin{array}{l}\text { Panama } \\
\text { City } \\
\text { Beaches } \\
\text { SPP }\end{array}$ & Bay & 1999 & $9,000,000$ & Offshore & 0.35 & 1.5 & 0 & 0.74 & $51 \%$ \\
\hline $\begin{array}{l}\text { Pinellas } \\
\text { County } \\
\text { Beach } \\
\text { Erosion } \\
\text { Control } \\
\text { Project } \\
\end{array}$ & Pinellas & 1999 & $2,612,166$ & $\begin{array}{c}\text { O\&M } \\
\text { Channel }\end{array}$ & & & 0.38 & & $94 \%$ \\
\hline \multirow{2}{*}{\begin{tabular}{|c|} 
Pinellas \\
County \\
Beach \\
Erosion \\
Control \\
Project \\
\end{tabular}} & \multirow[b]{2}{*}{ Pinellas } & \multirow[b]{2}{*}{2000} & 358,900 & \multirow{3}{*}{ Inlet } & \multirow{2}{*}{$\begin{array}{l}0.18 \\
0.18\end{array}$} & \multirow[t]{2}{*}{9} & 0.27 & 0.77 & $91 \%$ \\
\hline & & & 348,722 & & & & 0.33 & 0.83 & $91 \%$ \\
\hline $\begin{array}{c}\text { Brevard } \\
\text { SPP, North } \\
\text { Reach and } \\
\text { Patrick AFB }\end{array}$ & Brevard & 2001 & $3,740,800$ & & 0.33 & & 0.39 & 0.5 & $-67 \%$ \\
\hline $\begin{array}{c}\text { South } \\
\text { Amelia } \\
\text { Island } \\
\text { Beach } \\
\text { Nourishment }\end{array}$ & Nassau & 2002 & $1,864,489$ & Offshore & 0.21 & 1.82 & 0.3 & 0.87 & $52 \%$ \\
\hline $\begin{array}{l}\text { Manatee } \\
\text { SPP }\end{array}$ & N & 2002 & $1,900,000$ & Inlet & 0.24 & 2.44 & 0.25 & 3.3 & $-35 \%$ \\
\hline $\begin{array}{c}\text { Delray } \\
\text { Beach SPP } \\
\end{array}$ & $\mathrm{Pa}$ & 2002 & 123,000 & Offshore & 0.25 & 1.9 & 0.29 & 1.64 & $14 \%$ \\
\hline $\begin{array}{l}\text { Brevard } \\
\text { SPP }\end{array}$ & Brevard & 2003 & $1,504,005$ & Offshore & 0.33 & 1.2 & 0.36 & 0.6 & $50 \%$ \\
\hline $\begin{array}{l}\text { Indian River } \\
\text { Sector } 1 \& 2\end{array}$ & Indian River & 2003 & 589,350 & Offshore & 0.51 & 1.96 & 0.35 & 0.8 & $59 \%$ \\
\hline $\begin{array}{c}\text { Pensacola } \\
\text { Beach } \\
\text { Nourishment } \\
\end{array}$ & & 2003 & $4,248,300$ & Offshore & 0.33 & 0.85 & 0.35 & 0.6 & $29 \%$ \\
\hline $\begin{array}{l}\text { Lovers Key } \\
\text { Beach } \\
\text { Restoration }\end{array}$ & Lee & 2004 & 587,746 & Inlet & 0.16 & 5.49 & 0.26 & 1.69 & $69 \%$ \\
\hline $\begin{array}{l}\text { Pinellas } \\
\text { Beach } \\
\text { Erosion } \\
\text { Control } \\
\text { Project }\end{array}$ & Pinellas & 2004 & 412,255 & Inlet & 0.24 & 1.83 & 0.28 & 1.04 & $43 \%$ \\
\hline
\end{tabular}




\begin{tabular}{|c|c|c|c|c|c|c|c|c|c|}
\hline \multirow[b]{2}{*}{$\begin{array}{l}\text { Project } \\
\text { Name }\end{array}$} & \multirow[b]{2}{*}{ County } & \multirow[b]{2}{*}{ Year } & \multirow[b]{2}{*}{$\begin{array}{c}\text { Fill } \\
\text { Volume } \\
\text { (cy) }\end{array}$} & \multirow[b]{2}{*}{$\begin{array}{l}\text { Sediment } \\
\text { Source } \\
\end{array}$} & \multicolumn{2}{|c|}{$\begin{array}{l}\text { In Situ Sand } \\
\text { Source } \\
\text { Composite }\end{array}$} & \multicolumn{2}{|c|}{$\begin{array}{c}\text { Post-Fill Beach } \\
\text { Composite }\end{array}$} & \multirow[b]{2}{*}{$\begin{array}{l}\text { Percent } \\
\text { Change } \\
\text { in Fines }\end{array}$} \\
\hline & & & & & $\begin{array}{l}\text { Mean } \\
(\mathrm{mm})\end{array}$ & $\begin{array}{c}\% \\
\text { Passing } \\
\# 230\end{array}$ & $\begin{array}{l}\text { Mean } \\
(\mathrm{mm})\end{array}$ & \begin{tabular}{|c|}
$\%$ \\
Passing \\
$\# 230$
\end{tabular} & \\
\hline $\begin{array}{c}\text { Bonita } \\
\text { Beach } \\
\text { Restoration }\end{array}$ & Lee & 2004 & 145,000 & Inlet & 0.33 & 0.55 & 0.32 & 0.59 & $-7 \%$ \\
\hline $\begin{array}{l}\text { Brevard } \\
\text { SPP }\end{array}$ & Brevard & 2005 & $2,750,000$ & Offshore & 0.39 & & 0.37 & 0.4 & $-74 \%$ \\
\hline $\begin{array}{c}\text { Duval } \\
\text { Co.SPP }\end{array}$ & Duval & 2005 & 710,000 & Offshore & 0.25 & & 0.25 & 0.7 & $79 \%$ \\
\hline Martin SPP & Martin & 2005 & 885,000 & Offshore & 0.48 & 3 & $\mathrm{n} / \mathrm{a}$ & 1.17 & $61 \%$ \\
\hline $\begin{array}{c}\text { Panama } \\
\text { City }\end{array}$ & Bay & 2005 & $3,960,830$ & Offshore & 27 & & 0.3 & 0.62 & $33 \%$ \\
\hline $\begin{array}{l}\text { Lee SPP, } \\
\text { Captiva } \\
\text { Island }\end{array}$ & Lee & 2006 & $1,017,387$ & Offs & 8 & & 0.44 & 1.14 & $56 \%$ \\
\hline $\begin{array}{l}\text { Lee SPP, } \\
\text { Sanibel } \\
\text { Island }\end{array}$ & Lee & 2006 & 244,630 & & 0.38 & & 0.38 & 0.96 & $63 \%$ \\
\hline $\begin{array}{l}\text { Broward } \\
\text { SPP, } \\
\text { Segment III }\end{array}$ & Broward & 2006 & $1,794,700$ & & 0.4 & 6 & $\mathrm{n} / \mathrm{a}$ & 1.96 & $25 \%$ \\
\hline Collier BEC & Collier & 2006 & 667,562 & Offshore & 0.31 & 1.75 & 0.41 & 0.61 & $65 \%$ \\
\hline $\begin{array}{l}\text { Honeymoon } \\
\text { Island } \\
\text { Beach } \\
\text { Restoration } \\
\end{array}$ & & 2006 & 140,000 & & 0.2 & 3 & 0.16 & 0.05 & $98 \%$ \\
\hline $\begin{array}{c}\text { Navarre } \\
\text { Beach }\end{array}$ & $\mathrm{Sa}$ & 2006 & $2,952,899$ & Offshore & 0.4 & 0.3 & 0.32 & 0.28 & $7 \%$ \\
\hline $\begin{array}{c}\text { Pensacola } \\
\text { Beach }\end{array}$ & & 2006 & $3,159,264$ & Offshore & 0.33 & 1 & 0.37 & 0.9 & $10 \%$ \\
\hline $\begin{array}{c}\text { South Marco } \\
\text { Beach } \\
\text { Nourishment }\end{array}$ & Collier & 2006 & 176,000 & Inlet & 0.33 & 0.5 & $\mathrm{n} / \mathrm{a}$ & 0.6 & $-20 \%$ \\
\hline $\begin{array}{c}\text { Tampa } \\
\text { Harbor } \\
\text { O\&M, } \\
\text { Egmont Key }\end{array}$ & & 2006 & $1,329,000$ & $\begin{array}{c}\text { O\&M } \\
\text { Channel }\end{array}$ & 0.35 & 25 & 0.27 & 2.5 & $90 \%$ \\
\hline $\begin{array}{c}\text { Canaveral } \\
\text { Harbor - } \\
\text { Sand } \\
\text { Bypass } \\
\end{array}$ & Brevard & 2007 & 761,000 & Offshore & 0.18 & 4.4 & 0.18 & 1.3 & $70 \%$ \\
\hline $\begin{array}{l}\text { Ft. Pierce } \\
\text { SPP }\end{array}$ & St. Lucie & 2007 & 517,000 & Offshore & 0.43 & 1.6 & 0.6 & 0.1 & $94 \%$ \\
\hline $\begin{array}{l}\text { Kings Bay } \\
\text { Entrance } \\
\text { Channel }\end{array}$ & Nassau & 2007 & 80,000 & $\begin{array}{c}\text { O\&M } \\
\text { Channel }\end{array}$ & 0.39 & 1.9 & 0.33 & 1.4 & $26 \%$ \\
\hline
\end{tabular}




\begin{tabular}{|c|c|c|c|c|c|c|c|c|c|}
\hline \multirow[b]{2}{*}{$\begin{array}{l}\text { Project } \\
\text { Name }\end{array}$} & \multirow[b]{2}{*}{ County } & \multirow[b]{2}{*}{ Year } & \multirow[b]{2}{*}{$\begin{array}{c}\text { Fill } \\
\text { Volume } \\
\text { (cy) }\end{array}$} & \multirow[b]{2}{*}{$\begin{array}{l}\text { Sediment } \\
\text { Source }\end{array}$} & \multicolumn{2}{|c|}{$\begin{array}{c}\text { In Situ Sand } \\
\text { Source } \\
\text { Composite } \\
\end{array}$} & \multicolumn{2}{|c|}{$\begin{array}{l}\text { Post-Fill Beach } \\
\text { Composite }\end{array}$} & \multirow[b]{2}{*}{\begin{tabular}{|l} 
Percen \\
Change \\
in Fines
\end{tabular}} \\
\hline & & & & & $\begin{array}{l}\text { Mean } \\
(\mathrm{mm})\end{array}$ & \begin{tabular}{|c|}
$\%$ \\
Passing \\
$\# 230$ \\
\end{tabular} & $\begin{array}{l}\text { Mean } \\
(\mathrm{mm})\end{array}$ & \begin{tabular}{|c|}
$\%$ \\
Passing \\
$\# 230$ \\
\end{tabular} & \\
\hline $\begin{array}{c}\text { IWW O\&M, } \\
\text { Volusia }\end{array}$ & Volusia & 2008 & 569,000 & $\begin{array}{c}\text { O\&M } \\
\text { Channel }\end{array}$ & 0.18 & 1.7 & 0.19 & 0.8 & $53 \%$ \\
\hline $\begin{array}{c}\text { Lee SPP, } \\
\text { Captiva } \\
\text { Island } \\
\end{array}$ & Lee & 2008 & 98,270 & Offshore & 0.4 & & 0.51 & 0.53 & $39 \%$ \\
\hline Nassau SPP & Nassau & 2008 & $1,725,000$ & Offshore & n/a & & 0.25 & 0.7 & $65 \%$ \\
\hline $\begin{array}{l}\text { Ft. Pierce } \\
\text { SPP }\end{array}$ & St. Lucie & 2009 & 185,491 & Offshore & 0.43 & 1.6 & 0.46 & 1.46 & $9 \%$ \\
\hline Lido Key & Sarasota & 2009 & 536,000 & Inlet & 0.18 & 1.03 & 0.22 & 0.67 & $35 \%$ \\
\hline $\begin{array}{l}\text { Sanibel } \\
\text { Island }\end{array}$ & Lee & 2009 & 136,900 & Inlet & 0.38 & & 0.98 & 0.63 & $53 \%$ \\
\hline $\begin{array}{c}\text { Sailfish } \\
\text { Point }\end{array}$ & Martin & 2009 & 18,200 & Inlet & 0. & & 0.28 & 0.04 & $97 \%$ \\
\hline $\begin{array}{c}\text { Brevard } \\
\text { SPP, South } \\
\text { Reach }\end{array}$ & Brevard & 2010 & 111 & & 0.39 & & 0.41 & 0.48 & $-243 \%$ \\
\hline $\begin{array}{l}\text { Eglin AFB } \\
\text { Beach } \\
\text { Restoration }\end{array}$ & Okaloosa & 2010 & $2,700,000$ & & 0.33 & 3 & 0.36 & 0.04 & $97 \%$ \\
\hline $\begin{array}{c}\text { GIWW } \\
\text { O\&M, } \\
\text { Venice Inlet }\end{array}$ & & 2010 & & $\begin{array}{c}\text { O\&M } \\
\text { Channel }\end{array}$ & 0.16 & 1.69 & 0.22 & 0.21 & $88 \%$ \\
\hline $\begin{array}{c}\text { GIWW } \\
\text { O\&M, } \\
\text { John's Pass }\end{array}$ & & 2010 & & $\begin{array}{c}\text { O\&M } \\
\text { Channel }\end{array}$ & 0.24 & 0.86 & 0.22 & 0.21 & $76 \%$ \\
\hline $\begin{array}{c}\text { IWW O\&M, } \\
\text { Bakers } \\
\text { Haulover } \\
\text { Inlet }\end{array}$ & & 2010 & 000 & $\begin{array}{c}\text { O\&M } \\
\text { Channel }\end{array}$ & 0.26 & 6.48 & 0.67 & 0.2 & $97 \%$ \\
\hline $\begin{array}{c}\text { North Boca } \\
\text { Raton }\end{array}$ & Palm Beach & 2010 & 782,200 & Offshore & 0.27 & 1.82 & 0.31 & 0.35 & $81 \%$ \\
\hline $\begin{array}{l}\text { St. Joseph } \\
\text { Peninsula } \\
\text { Beach } \\
\text { Restoration }\end{array}$ & & 2010 & $3,600,000$ & Offshore & 0.33 & 1 & 0.28 & 1 & $0 \%$ \\
\hline $\begin{array}{c}\text { Treasure } \\
\text { Island/ Long } \\
\text { Key SPP }\end{array}$ & Pinellas & 2010 & 160,000 & $\begin{array}{c}\text { O\&M } \\
\text { Channel }\end{array}$ & 0.24 & 1.71 & 0.18 & 0.21 & $88 \%$ \\
\hline $\begin{array}{l}\text { Anna Maria } \\
\text { Island }\end{array}$ & Manatee & 2011 & 24,700 & Inlet & 0.21 & 1.86 & 0.24 & 0.64 & $66 \%$ \\
\hline $\begin{array}{l}\text { Coquina } \\
\text { Beach }\end{array}$ & Manatee & 2011 & 204,800 & Inlet & 0.21 & 1.86 & 0.21 & 1.13 & $39 \%$ \\
\hline
\end{tabular}




\begin{tabular}{|c|c|c|c|c|c|c|c|c|c|}
\hline \multirow[b]{2}{*}{$\begin{array}{l}\text { Project } \\
\text { Name }\end{array}$} & \multirow[b]{2}{*}{ County } & \multirow[b]{2}{*}{ Year } & \multirow[b]{2}{*}{$\begin{array}{c}\text { Fill } \\
\text { Volume } \\
\text { (cy) }\end{array}$} & \multirow[b]{2}{*}{$\begin{array}{l}\text { Sediment } \\
\text { Source }\end{array}$} & \multicolumn{2}{|c|}{$\begin{array}{l}\text { In Situ Sand } \\
\text { Source } \\
\text { Composite }\end{array}$} & \multicolumn{2}{|c|}{$\begin{array}{l}\text { Post-Fill Beach } \\
\text { Composite }\end{array}$} & \multirow[b]{2}{*}{$\begin{array}{l}\text { Percent } \\
\text { Change } \\
\text { in Fines }\end{array}$} \\
\hline & & & & & $\begin{array}{l}\text { Mean } \\
(\mathrm{mm})\end{array}$ & $\begin{array}{c}\% \\
\text { Passing } \\
\# 230\end{array}$ & $\begin{array}{l}\text { Mean } \\
(\mathrm{mm})\end{array}$ & $\begin{array}{c}\% \\
\text { Passing } \\
\# 230\end{array}$ & \\
\hline $\begin{array}{c}\text { Dade BEC, } \\
\text { Miami } \\
\text { Beach (CT- } \\
\text { E) }\end{array}$ & Miami-Dade & 2011 & 206,042 & Offshore & 0.42 & 2.86 & 0 & 0.35 & $88 \%$ \\
\hline $\begin{array}{c}\text { Duval } \\
\text { Co.SPP }\end{array}$ & Duval & 2011 & 689,015 & Offshore & 0.26 & & 0.25 & 0.7 & $78 \%$ \\
\hline $\begin{array}{l}\text { Ft. Pierce } \\
\text { SPP }\end{array}$ & St. Lucie & 2011 & 431,301 & Offshore & 0.58 & 1 & 0.5 & 0.19 & $91 \%$ \\
\hline \begin{tabular}{|c|} 
Hideaway \\
Beach \\
Nourishment
\end{tabular} & Collier & 2011 & 129,705 & Nearsh & 0.37 & & 0.32 & 0.65 & $58 \%$ \\
\hline \begin{tabular}{|c|} 
IWW J-M \\
O\&M, \\
Mantanazas \\
Inlet
\end{tabular} & St. Johns & 2011 & 288,647 & $\begin{array}{c}\text { O\&M } \\
\text { Channel }\end{array}$ & 0.16 & & 0.24 & 0.29 & $91 \%$ \\
\hline \begin{tabular}{|c|} 
Panama \\
City Beach, \\
\end{tabular} & Bay & 2011 & $1,370,636$ & Of & 0.25 & 0.94 & 0.36 & 0.51 & $46 \%$ \\
\hline $\begin{array}{c}\text { Pinellas } \\
\text { SPP, Sand } \\
\text { Key }\end{array}$ & Pinellas & 2011 & $1,200,000$ & & 0.18 & 3.04 & 0.28 & 0.58 & $81 \%$ \\
\hline $\begin{array}{l}\text { South } \\
\text { Amelia } \\
\text { Island }\end{array}$ & & 2011 & $1,999,245$ & & 0.25 & 1.07 & 0.48 & 0.43 & $60 \%$ \\
\hline $\begin{array}{l}\text { Dade BEC, } \\
\text { Miami } \\
\text { Beach (CT- } \\
\text { E) }\end{array}$ & $\mathrm{Mi}$ & 2012 & 77,237 & Inlet & 0.51 & 0.99 & 0.52 & 0.27 & $73 \%$ \\
\hline $\begin{array}{l}\text { IWW St. } \\
\text { Aug Inlet }\end{array}$ & 0 & 2012 & 122,648 & $\begin{array}{c}\text { O\&M } \\
\text { Channel }\end{array}$ & 0.28 & 2.57 & 0.28 & 0.41 & $84 \%$ \\
\hline $\begin{array}{l}\text { Palm Beach } \\
\text { Harbor O\&M }\end{array}$ & Palm Beach & 2012 & 424,819 & $\begin{array}{c}\text { O\&M } \\
\text { Channel }\end{array}$ & 0.28 & 3.66 & 0.36 & 0.27 & $93 \%$ \\
\hline $\begin{array}{l}\text { St. Johns } \\
\text { SPP }\end{array}$ & St. Johns & 2012 & $2,199,340$ & Inlet & 0.23 & 1.43 & 0.33 & 0.28 & $80 \%$ \\
\hline $\begin{array}{l}\text { AIWW } \\
\text { Sawpit }\end{array}$ & & 2013 & 550,000 & $\begin{array}{c}\text { O\&M } \\
\text { Channel }\end{array}$ & 0.17 & 1.72 & 0.18 & 1.2 & $30 \%$ \\
\hline $\begin{array}{l}\text { Brevard } \\
\text { SPP, North } \\
\text { Reach }\end{array}$ & Brevard & 2013 & 692,418 & Offshore & 0.3 & 5.5 & 0.35 & 0.59 & $89 \%$ \\
\hline $\begin{array}{c}\text { Brevard } \\
\text { SPP, South } \\
\text { Reach }\end{array}$ & Brevard & 2013 & 972,410 & Offshore & 0.27 & 0.11 & 0.45 & 0.02 & $82 \%$ \\
\hline $\begin{array}{c}\text { Dade BEC, } \\
\text { Bal Harbor } \\
\text { (CT-G) }\end{array}$ & Miami-Dade & 2013 & 236,000 & Inlet & 0.42 & 2.2 & 0.41 & 0.11 & $95 \%$ \\
\hline
\end{tabular}


ERDC/CHL CHETN-VI-?

February 2019

\begin{tabular}{|c|c|c|c|c|c|c|c|c|c|}
\hline \multirow[b]{2}{*}{$\begin{array}{l}\text { Project } \\
\text { Name }\end{array}$} & \multirow[b]{2}{*}{ County } & \multirow[b]{2}{*}{ Year } & \multirow[b]{2}{*}{$\begin{array}{c}\text { Fill } \\
\text { Volume } \\
\text { (cy) }\end{array}$} & \multirow[b]{2}{*}{$\begin{array}{l}\text { Sediment } \\
\text { Source }\end{array}$} & \multicolumn{2}{|c|}{$\begin{array}{c}\text { In Situ Sand } \\
\text { Source } \\
\text { Composite } \\
\end{array}$} & \multicolumn{2}{|c|}{$\begin{array}{l}\text { Post-Fill Beach } \\
\text { Composite }\end{array}$} & \multirow[b]{2}{*}{\begin{tabular}{|l} 
Percen \\
Change \\
in Fines
\end{tabular}} \\
\hline & & & & & $\begin{array}{l}\text { Mean } \\
(\mathrm{mm})\end{array}$ & \begin{tabular}{|c|}
$\%$ \\
Passing \\
$\# 230$ \\
\end{tabular} & $\begin{array}{l}\text { Mean } \\
(\mathrm{mm})\end{array}$ & \begin{tabular}{|c||}
$\%$ \\
Passing \\
$\# 230$ \\
\end{tabular} & \\
\hline $\begin{array}{l}\text { Ft. Pierce } \\
\text { O\&M }\end{array}$ & St. Lucie & 2013 & 164,100 & Inlet & 0.49 & 1.35 & 0.46 & 1.2 & $11 \%$ \\
\hline $\begin{array}{l}\text { Ft. Pierce } \\
\text { SPP }\end{array}$ & St. Lucie & 2013 & 430,000 & Offshore & 0.43 & 1.6 & 0.48 & 0.28 & $83 \%$ \\
\hline $\begin{array}{l}\text { Hobe Sound } \\
\text { O\&M }\end{array}$ & Martin & 2013 & 2,760 & $\begin{array}{c}\text { O\&M } \\
\text { Channel }\end{array}$ & 0.44 & & 0.48 & 1.21 & $28 \%$ \\
\hline $\begin{array}{c}\text { IWW Bakers } \\
\text { Haulover } \\
\text { O\&M }\end{array}$ & Miami-Dade & 2013 & 49,592 & $\begin{array}{c}\text { O\&M } \\
\text { Channel }\end{array}$ & 0.26 & 8.32 & 0.49 & & $98 \%$ \\
\hline $\begin{array}{l}\text { IWW Jupiter } \\
\text { O\&M }\end{array}$ & Palm Beach & 2013 & 88,986 & $\begin{array}{c}\text { O\&M } \\
\text { Channel }\end{array}$ & 0.28 & 1.26 & 0.27 & 0.49 & $61 \%$ \\
\hline $\begin{array}{l}\text { Lee SPP, } \\
\text { Gasparilla }\end{array}$ & Lee & 2013 & 461,676 & Offshore & 0.29 & 2.2 & 0.47 & 0.33 & $85 \%$ \\
\hline $\begin{array}{c}\text { Manatee } \\
\text { SPP, Anna } \\
\text { Maria Island }\end{array}$ & Manatee & 2013 & 887,948 & 0 & 0.27 & & 0.37 & 0.32 & $73 \%$ \\
\hline Martin SPP & Martin & 2013 & 613,017 & Offshore & 0.39 & 2.47 & 0.48 & 0.28 & $89 \%$ \\
\hline $\begin{array}{c}\text { Palm Beach } \\
\text { SPP }\end{array}$ & Palm Beach & 2013 & 88,986 & $\begin{array}{c}\text { O\&M } \\
\text { Channel }\end{array}$ & 0.28 & 1.26 & 1.89 & 0.49 & $61 \%$ \\
\hline $\begin{array}{c}\text { Palm Beach } \\
\text { SPP, Delray } \\
\text { Beach }\end{array}$ & Palm & 2013 & 30,189 & re & 0.24 & 0.98 & 0.28 & 0.24 & $76 \%$ \\
\hline $\begin{array}{l}\text { Palm Beach } \\
\text { SPP, N. } \\
\text { Boca }\end{array}$ & $\mathrm{Pal}$ & 2013 & 041 & Offshore & 0.28 & 1.82 & 0.32 & 0.43 & $76 \%$ \\
\hline $\begin{array}{c}\text { Palm Beach } \\
\text { SPP, Ocean } \\
\text { Ridge }\end{array}$ & Palm B & 2013 & 503,691 & Offshore & 0.24 & 1.28 & 0.34 & 0.27 & $79 \%$ \\
\hline $\begin{array}{c}\text { S. Lake } \\
\text { Worth Inlet } \\
\text { O\&M }\end{array}$ & Palm Beach & 2013 & Unknown & Inlet & 0.25 & 1.23 & 0.45 & 0.02 & $98 \%$ \\
\hline $\begin{array}{l}\text { St Augustine } \\
\text { Harbor O\&M }\end{array}$ & Ct lohno & 2013 & 184,000 & $\begin{array}{c}\text { O\&M } \\
\text { Channel }\end{array}$ & 0.57 & 1.98 & 0.45 & 0.6 & $70 \%$ \\
\hline $\begin{array}{l}\text { St Augustine } \\
\text { Harbor O\&M }\end{array}$ & & 2013 & 184,000 & $\begin{array}{c}\text { O\&M } \\
\text { Channel }\end{array}$ & 0.29 & 1.29 & 0.45 & 0.6 & $53 \%$ \\
\hline $\begin{array}{c}\text { Treasure } \\
\text { Island/ Long } \\
\text { Key SPP }\end{array}$ & Pinellas & 2013 & 249,164 & Offshore & 0.24 & 1.83 & 0.28 & 0.3 & $84 \%$ \\
\hline $\begin{array}{c}\text { Wiggins } \\
\text { Pass O\&M }\end{array}$ & Collier & 2013 & 107,370 & Inlet & 0.36 & 2.42 & 0.66 & 0.48 & $80 \%$ \\
\hline $\begin{array}{c}\text { Brevard } \\
\text { Co.SPP, } \\
\text { South } \\
\text { Reach }\end{array}$ & Brevard & 2014 & 692,418 & Offshore & 0.33 & 0.3 & 0.45 & 0 & $100 \%$ \\
\hline
\end{tabular}




\begin{tabular}{|c|c|c|c|c|c|c|c|c|c|}
\hline \multirow[b]{2}{*}{$\begin{array}{l}\text { Project } \\
\text { Name } \\
\end{array}$} & \multirow[b]{2}{*}{ County } & \multirow[b]{2}{*}{ Year } & \multirow[b]{2}{*}{$\begin{array}{c}\text { Fill } \\
\text { Volume } \\
\text { (cy) } \\
\end{array}$} & \multirow[b]{2}{*}{$\begin{array}{l}\text { Sediment } \\
\text { Source }\end{array}$} & \multicolumn{2}{|c|}{$\begin{array}{l}\text { In Situ Sand } \\
\text { Source } \\
\text { Composite }\end{array}$} & \multicolumn{2}{|c|}{$\begin{array}{c}\text { Post-Fill Beach } \\
\text { Composite }\end{array}$} & \multirow[b]{2}{*}{$\begin{array}{l}\text { Percent } \\
\text { Change } \\
\text { in Fines }\end{array}$} \\
\hline & & & & & \begin{tabular}{|l} 
Mean \\
$(\mathrm{mm})$
\end{tabular} & \begin{tabular}{|c|}
$\%$ \\
Passing \\
$\# 230$ \\
\end{tabular} & $\begin{array}{l}\text { Mean } \\
(\mathrm{mm})\end{array}$ & \begin{tabular}{|c}
$\%$ \\
Passing \\
\#230
\end{tabular} & \\
\hline $\begin{array}{l}\text { Palm Beach } \\
\text { Co. Delray } \\
\text { Beach SPP }\end{array}$ & Palm Beach & 2014 & 675,000 & Offshore & 0.23 & 0.89 & & 0.59 & $34 \%$ \\
\hline $\begin{array}{l}\text { Palm Beach } \\
\text { Co. Ocean } \\
\text { Ridge SPP }\end{array}$ & Palm Beach & 2014 & 503,690 & Offshore & 0.24 & & & 0.01 & $99 \%$ \\
\hline $\begin{array}{l}\text { Palm Beach } \\
\text { SPP, } \\
\text { Jupiter- } \\
\text { Carlin }\end{array}$ & Palm Beach & 2014 & 132,638 & Offshore & & 6 & 0.52 & & $79 \%$ \\
\hline $\begin{array}{c}\text { Sailfish } \\
\text { Point O\&M }\end{array}$ & Martin & 2014 & 25,000 & $\ln \mid$ & $\mathrm{n} / \mathrm{a}$ & & 0.34 & 0.18 & $81 \%$ \\
\hline $\begin{array}{c}\text { Tampa } \\
\text { Harbor } \\
\text { O\&M, } \\
\text { Egmont Key }\end{array}$ & Hillsborough & 2014 & 319,712 & ( & & & 0.37 & 1.53 & $94 \%$ \\
\hline $\begin{array}{c}\text { Venice } \\
\text { Beach SPP }\end{array}$ & Sarasota & 2014 & 719,917 & & 0.43 & 1.95 & $\mathrm{n} / \mathrm{a}$ & 0.31 & $84 \%$ \\
\hline $\begin{array}{c}\text { East Pass } \\
\text { O\&M }\end{array}$ & Okaloosa & 2015 & 40,000 & & 0.3 & 0.26 & 0.32 & 0.1 & $62 \%$ \\
\hline $\begin{array}{l}\text { Ft. Pierce } \\
\text { SPP }\end{array}$ & St. Lu & 2015 & 319,091 & Offshore & 0.43 & 1.6 & 0.47 & 0.21 & $87 \%$ \\
\hline $\begin{array}{c}\text { Bonita } \\
\text { Beach/ } \\
\text { Lovers Key }\end{array}$ & & 15 & 342,400 & Offshore & 0.27 & 0.55 & 0.37 & 0.3 & $45 \%$ \\
\hline \multirow{5}{*}{ Composite } & \multicolumn{4}{|c|}{ Average: } & 0.31 & 2.64 & 0.37 & 0.74 & $54 \%$ \\
\hline & \multicolumn{4}{|c|}{ Median: } & 0.29 & 1.75 & 0.34 & 0.51 & $70 \%$ \\
\hline & \multicolumn{4}{|c|}{ Standard Deviation: } & 0.10 & 3.60 & 0.20 & 0.81 & $50 \%$ \\
\hline & \multicolumn{4}{|c|}{ Minimum: } & 0.14 & 0.11 & 0.11 & 0.00 & $-243 \%$ \\
\hline & \multicolumn{4}{|c|}{ Maximum: } & 0.58 & 25.00 & 1.89 & 5.30 & $100 \%$ \\
\hline
\end{tabular}

The relationship between the changes in the percent fines from the in situ sand source to the post-construction beach is shown in Figure 3. The majority of the data points is clustered between $0 \%-4 \%$ fines for in situ percentages and $0 \%-1 \%$ fines for post-construction beaches; there is no overall trend to the data set except that fines were usually lost during the dredging process. Due to regulatory constraints, there is a lack of data in the historic project files for in situ material containing higher fines content. Only one project in the dataset was granted a waiver to the requirement of having a sand source with 5\% silt or less: Tampa Harbor O\&M, Egmont Key. This project placed dredged material from the Tampa Harbor Entrance Channel on Egmont Key State Park to protect both archaeological interests and turtle nesting habitat in 2006 and 2014. The sediments from the Tampa Harbor Entrance Channel with average in situ fines contents of $25 \%$ and $23.8 \%$, respectively, for different events were placed on the island's beach. 
The post-construction beach samples contained $2.5 \%$ and $1.53 \%$ fines, respectively. At Egmont Key, the dredging process resulted in a loss of $90 \%$ of the fines in the 2006 event and $94 \%$ of the fines during the 2014 event.

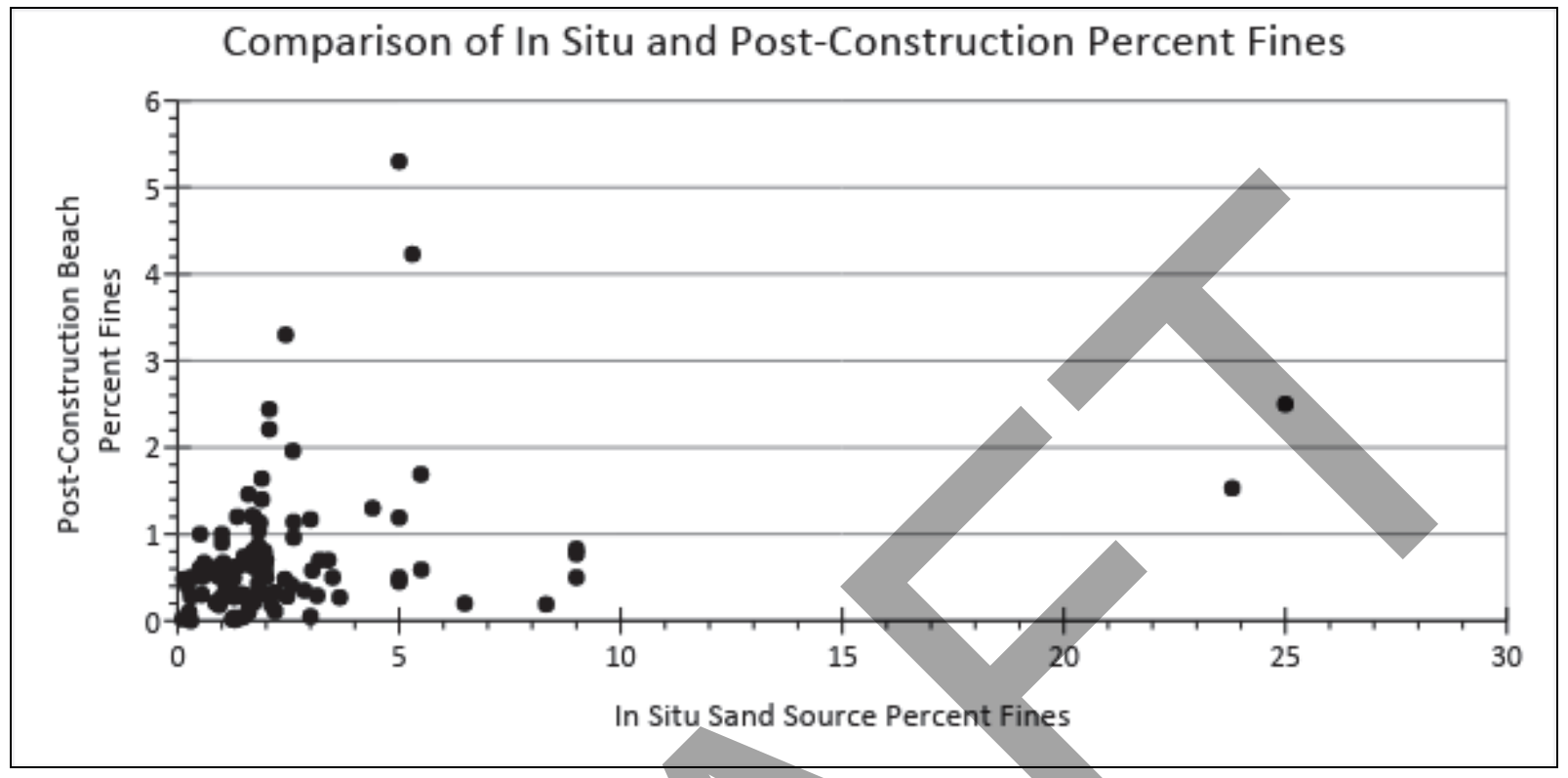

Figure 3. Summary of the loss of the composite percent fines from the in situ sand source to the postconstruction beach. Note scale is not 1:1.

In order to quantify the change in fines content, the percent change in fines content between in situ sand source sediments and post-construction beach sediments for a given project or construction event was found. A histogram was created from this data (Figure 4). The data set contains 103 placement events, of which $69 \%$ show a decrease of at least $50 \%$ fines from the in situ sand source. The mean and median percentages of the change in fines content are $57 \%$ and $70 \%$, respectively (Table 1). Additionally, 92 of the projects, or $90 \%$ of the data set, showed fines were lost to the system during the dredging process. 


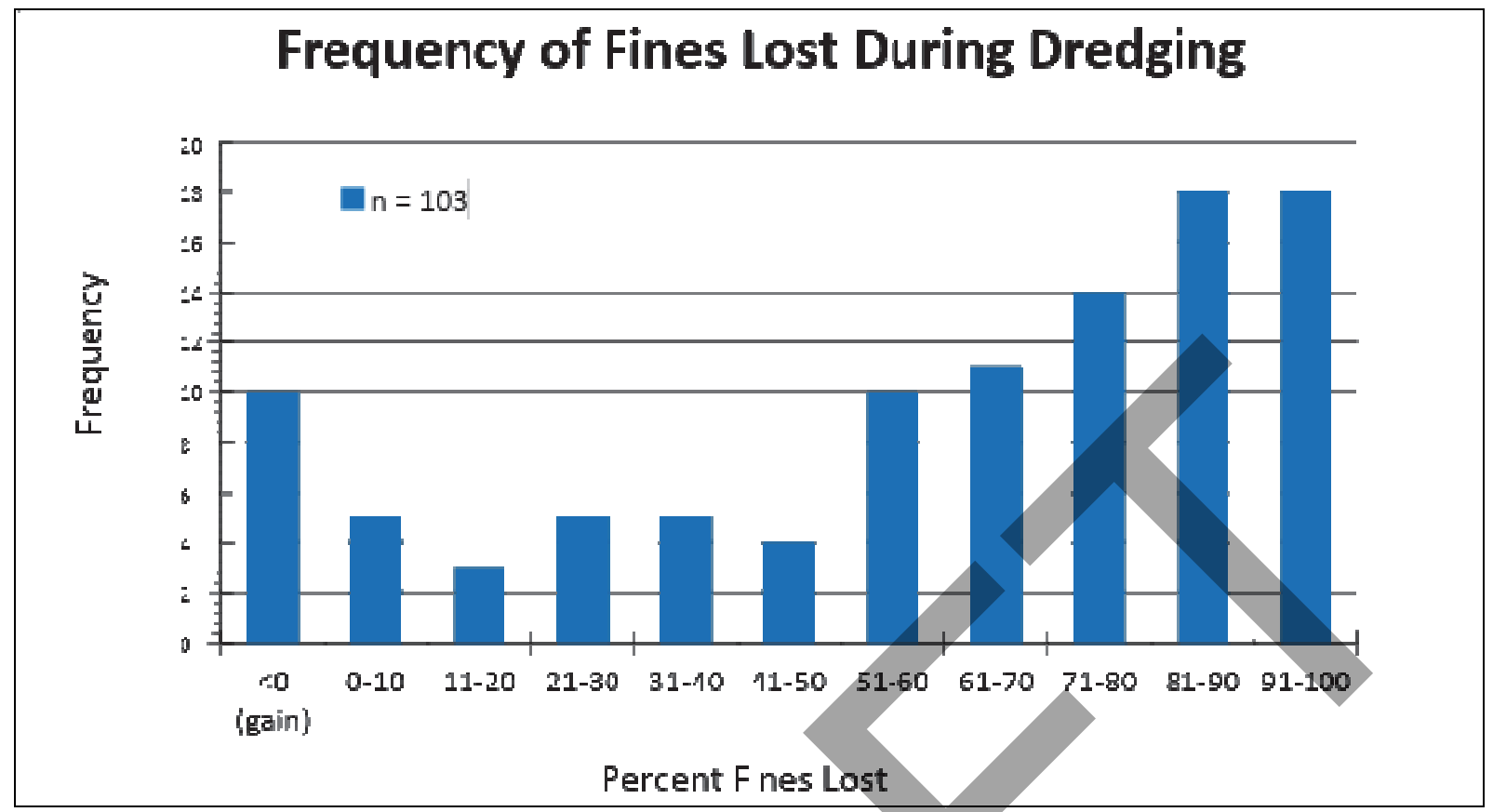

Figure 4. Frequency of fines loss for the data set.

There were 10 projects that did not lose fines during dredging and beach placement: North Boca (1988), Broward SPP (1991), Delray (1992), Sanibel Gulf Pines (1996), Captiva Island (1997), Brevard SPP (2001), Manatee SPP (2002), Bonita Beach (2004), Brevard SPP (2005), and South Marco (2006). In each of these cases, the difference between the percent silt of the in situ sand source and the post-construction beach was less than $1 \%$, averaging $0.27 \%$ and ranging from $0.04 \%$ to $0.86 \%$ difference. While this study's metrics do show there is an increase in fines from the in situ sand source to the post-construction beach during these 10 projects, the increase is negligible and never exceeds $1 \%$.

Potential Applications of Fines Loss Data. The data summarized herein, and available for use, are applicable in many coastal and navigation projects. A specific application of this dataset is to improve the planning-level selection process for suitable sand sources for beach nourishment. When obtaining a permit for beach placement in Florida, the FDEP regulators assume $0 \%$ reduction in fines content between the sand source and beach placement site. However, a zero fines loss scenario is known to be inaccurate based on the requirement for turbidity monitoring during construction. This data set shows that $69 \%$ of the projects experienced a loss of more than $50 \%$ fines, while $90 \%$ of the projects experienced some level of reduction in fines during the dredging process. This suggests that regulators could reasonably assume that similar projects would lose fines from the sand source to the beach, with a $50 \%$ loss of fines being a conservative estimate.

Note that no change is needed to the FDEP Sand Rule, only the application which places compliance criteria on the post-construction fill, understanding that it has changed from the in situ sediment source. Permitting sediment sources with a higher fines content both for beach nourishment and for beneficial placement from navigational channels will reduce the need for expansion of offshore dredge material disposal sites and upland dredge material management 
areas, increase the sediment available for beneficial use, allow more sediment sources to be used, warrant re-evaluation of existing sediment sources for a greater sediment yield, and offer additional Regional Sediment Management (RSM) opportunities. Results of this initiative are not only important to RSM goals, but information related to the sorting of sediment during dredging and the natural sorting of sediment once placed is key to the principles of Engineering With Nature.

Future Studies on Fines Loss. Future research will need to focus on refining thresholds for fines loss. A comparison of fines loss between hydraulic dredge types was beyond the scope of this study. Work is in progress with the Bureau of Ocean Energy Management (BOEM) to determine the percentage of fines lost between the dredge intake and pump-out as a part of the next phase in the series of studies on delivering sediment from the sand source to the placement site. These resulting data sets may be used to develop a function to predict the reduction in fines on the post-construction nourished beach from an in situ sand source.

Understanding the behavior and long-term fate of fine material provides an opportunity to better manage valuable sand resources while not endangering the environment.

SUMMARY: The data presented in this CHETN show an average change of 54\% in fines content occurs during the dredging process between composite values of the in situ sand source and the post-construction beach. The goal of this study was to empirically quantify the amount of fines change during dredging and placement events over time, increasing the baseline limits permissible for material that can be placed for beneficial use.

This research effort could benefit RSM strategies by maintaining a greater mass of sediment in the littoral zone instead of offshore or in upland placement and could reduce cost due to decreased pumping and transit distances. This would maximize the amount of sediment retained in the littoral system and eliminate the irreversible loss of littoral sediment that is currently not retained while providing reasonable assurance to regulating agencies of compliance with postconstruction percent fines criteria.

POINTS OF CONTACT: This Coastal and Hydraulics Engineering Technical Note (CHETN) was prepared as part of the USACE National Regional Sediment Management (RSM) Program and the USACE Dredging Operations and Environmental Research (DOER) Program by the U.S. Army Engineer Research and Development Center (ERDC), Coastal and Hydraulics Laboratory (CHL), and Environmental Laboratory (EL), respectively, Vicksburg, MS. Additional information regarding the RSM Program may be obtained from the RSM website (http://rsm.usace.army.mil) or from the USACE National RSM Program Manager, Dr. Katherine E. Brutsché (Katherine.E.Brutshce@usace.army.mil). Additional information regarding the DOER Program may be obtained from the DOER website (https://doer.el.erdc.dren.mil) or from the USACE DOER Program Manager, Dr. Todd S. Bridges (Todd.S.Bridges@usace.army.mil). Questions regarding this CHETN can be addressed to Dr. Jennifer Coor (904) 232-1054 (Jennifer.L.Coor@usace.army.mil) at USACE District, Jacksonville (SAJ). This document should be referenced as follows: 
Coor, J. L., and J. D. Ousley. 2019. Historical Analysis of the Change in Fines during Beach Nourishment. ERDC/CHL CHETN-VI-xx. Vicksburg, MS: U.S. Army Engineer Research and Development Center. <Library: Please insert DOI number $>$

\section{REFERENCES}

Clark, R. R. 1993. Beach conditions in Florida: A statewide inventory and identification of the beach erosion problem areas in Florida. Beaches and Shores Technical and Design Memorandum no. 89-1. Tallahassee, FL: Florida Department of Environmental Protection.

Coor, J. L., C. Beauvais, and J. D. Ousley. 2015. ROSS/OSSI (ROSSI): A coastal management tool for offshore sand sources. Proceedings of Coastal Sediments 2015. 265-276.

Dean, R. G., and R. A. Dalrymple. 2002. Coastal Processes with Engineering Applications. Cambridge, UK: Cambridge University Press.

Florida Department of Environmental Protection (FDEP). 2016a. Critically Eroded Beaches in Florida. Tallahassee, FL.

FDEP. 2016b. http://www.dep.state.fl.us/beaches

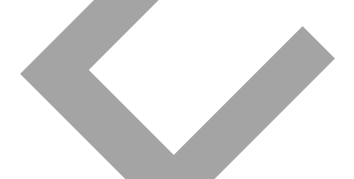

Friedman, G. M., and F. E. Sanders. 1978. Principles of Sedimentology. https://doi.org/10.1002/esp.3290040317

Ousley, J. D., E. Kromhout, M. H. Schrader, and L. Lillycrop. 2014. Southeast Florida Sediment Assessment and Needs Determination (SAND) Study: Final Report. ERDC/CHL TR-14-10. Vicksburg, MS: U.S. Army Engineer Research and Development Center.

Tanner, W. F. 1995. Environmental Clastic Granulometry. Florida Geological Survey Special Publication 40. Tallahassee, Florida.

U.S. Army Corps of Engineers (USACE). 2001. Coastal Engineering Manual. Part V, 22-28. Vicksburg, MS: U.S. Army Engineer Waterways Experiment Station.

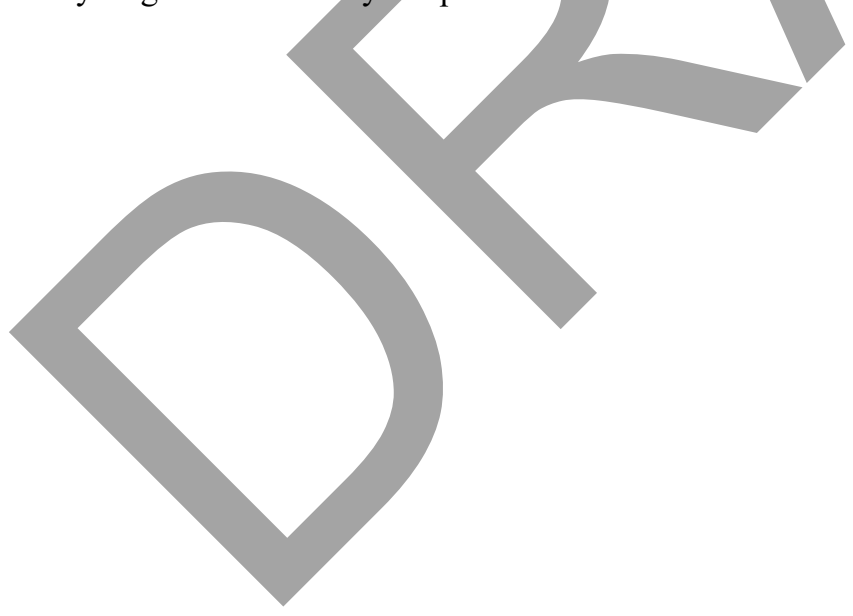

NOTE: The contents of this technical note are not to be used for advertising, publication, or promotional purposes. Citation of trade names does not constitute an official endorsement or approval of the use of such products. 